\title{
Insulin shot dependent lipodystrophy: evidence, uncertainties and current terminology overlaps
}

\begin{abstract}
Lipodystrophy (LD) is still an ill-defined pathological entity, common to a series of diseases including diabetes. Lipohypertrophy (LH) is its most common expression and depends mostly on repeated needle trauma to the skin in insulin treated patients. This short review tries to clarify some clinically relevant points and stresses the need to put a stronger effort on patient education and health care training in order to prevent any life-threatening hypoglycemic events eventually related to miss LH identification in people with diabetes.
\end{abstract}

Keywords: diabetes, lipodystrophy, terminology, methodology, lipohypertrophy, insulin-treated patients, trauma, low cultural level

\author{
Volume 3 Issue 3 - 2016 \\ Sandro Gentile, ' Antonio Ceriello, 2,3 Felice \\ Strollo ${ }^{4}$ on behalf of the AMD-OSDI Italian \\ Injection Technique Study Group \\ 'Department of Clinical and Experimental Medicine, Second \\ University of Naples, Europe \\ ${ }^{2}$ Department of Endocrinology, Hospital Clinic de Barcelona, \\ Europe \\ ${ }^{3}$ Centro de Investigacion Biomèdica en Red de Diabetes y \\ Enfermedades Metabolicas Asociadas (CIBERDEM), Barcelona, \\ Europe \\ ${ }^{4}$ Department of Endocrinology, Endocrinology and Metabolism \\ Unit, Europe
}

Correspondence: Felice Strollo, Elle-Di, Endocrinology and Metabolism Unit, Europe, Tel +390632196 39, Email felix.strollo@gmail.com

*AMD is the Italian acronym for the Association of Diabetes Specialists and OSDI is the Italian acronym for Diabetes Care Health Professionals

Received: March 29, 2016 | Published: May 03, 2016

\section{Mini review}

Cutaneous lipodystrophy (LD) has been known for decade's ad many authors described LD in insulin-treated patients. Lipohypertrophy (LH) is well documented to be its most frequent form as opposed to lipoatrophy (LA). ${ }^{1,2}$

A large body of evidence supports that LH is significantly associated to a series of parameters including female sex, low cultural level, high BMI, long duration of both disease and insulin treatment, use of long needles and, most of all, missed injection site rotation or repeated needle re-use. This topic is of great clinical interest indeed and, based on the evidence from 300 relevant related publications, was examined in depth by 150 top specialists from 58 countries at the Forum for Injection Technique \& Therapy Expert Recommendations (FITTER), an international meeting held in Rome in October $2015 .^{3}$ At the end of three intensive working days during which all delegates exchanged their experienced knowledge in the field, several recommendations were issued and have just been submitted for publication on an international scientific journal. Nevertheless a wide variation has been reported in the literature concerning LH frequency, ranging in fact from $28 \%$ to $67 \%,{ }^{1,2}$ probably depending on the fact that a thorough skin examination is often omitted in diabetes clinics.

However it should be pointed out that such variability may also be due to different levels of experience attained by involved health professionals (HPs), as well as, to the lack of a clearly defined, validated methodology devoted to LH identification. In fact, morphological features of such lesions are extremely variable in size, texture and protrusion above the skin. ${ }^{4}$ The hereby attached Figure 1 refers to a some of our patients with diabetes and ultrasound-ascertained LH undergoing blind palpation-based examination by four well trained
(WT) and as many non-trained (NT) HPs. It provides evidence of striking differences in the diagnostic ability between WT and NT HPs, being influenced primarily by training level and by individual LD morphological features as well.
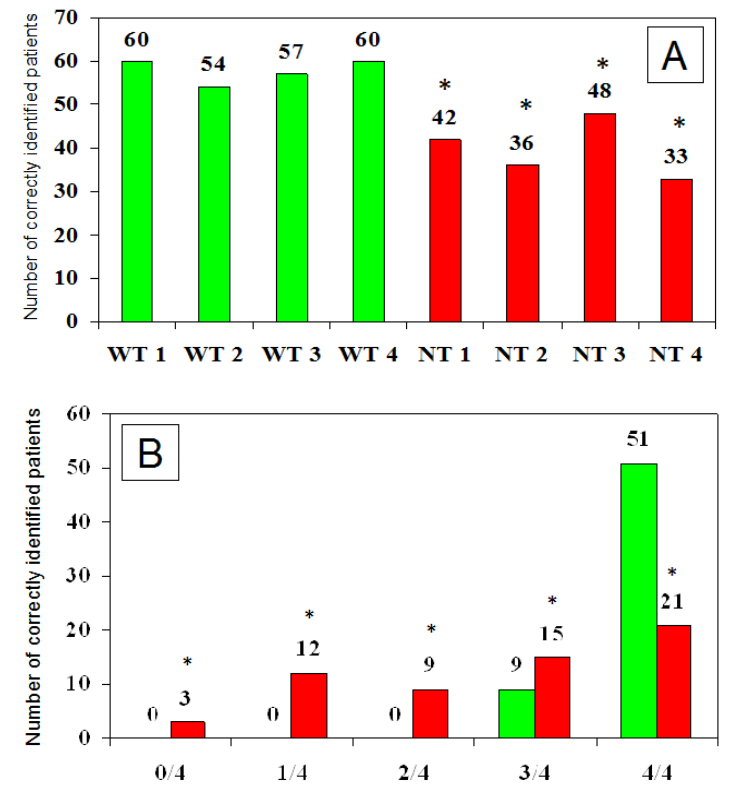

Figure I Number of patients identified as having LH by well trained (WT) or non-trained (NT) healthcare professionals (panel A) and number of well trained (green columns) or non-trained (red columns) health professionals, among 4 in each group, accurately identifying LH lesions (panel B) (*NT vs WT $\mathrm{p}<0.0 \mathrm{I})$. 
Missed LD identification may have relevant clinical consequences. In fact, when injected into LD areas, insulin causes wide glycemic oscillations including inappropriately high glucose levels and a high rate of unexplained hypoglycemic episodes, both of which being hardly responsive even to large dosage changes. ${ }^{2,5}$ Since educational activity on proper injection techniques in LD-affected individuals has proven effective in significantly reducing glucose oscillations, ${ }^{6}$ we suggest to look for lesions in all insulin treated patients, or at least to so systematically in those displaying repeated unexplained hypoglycemic events and/or wide glycemic variability. Despite the occasional improper use of term LH and LA as synonyms, ${ }^{1}$ we suggest to keep the two concepts separated. In fact LA is a scarring lesion characterized by the atrophy of subcutaneous fat tissue with loss of adipocytes and therefore is quite different from LH in terms of both morphology and pathogenesis (Figure 2). On the other hand LH is characterized by enlarged adipocytes within an area of swollen and/ or stiff fat tissue.?

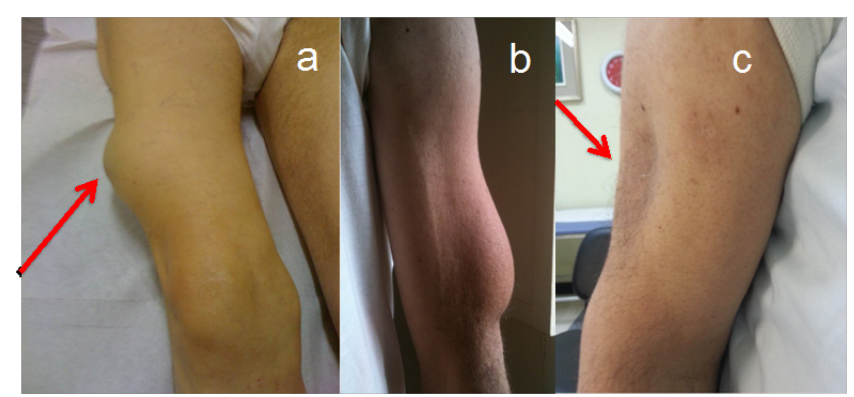

Figure $\mathbf{2} \mathrm{LH}$ lesion of the right thigh as shown by the arrow (panel A) and left arm (panel B); LA lesion of the left arm as shown by the arrow (panel C).

From a histopathological point of view, LH lesions are entirely contributed to by hypertrophic adipocytes (mostly two to three times larger than normal) which often invade the adjacent reticular dermis, are engulfed by lipid droplets, proliferate or display other signs of metabolic activation. ${ }^{8}$

Indeed, LH has been acknowledged as the consequence of mechanical stresses, such as those coming from needle re-use or missed injection site rotation, ${ }^{2}$ as well as, of inner insulin molecule growth promoting effects. ${ }^{5}$ Conversely, LA seems to be mostly associated with individual immuno-allergic and inflammatory factors ${ }^{2}$ and/or with poorly purified insulin preparations. ${ }^{6}$ This distinction is especially important from a clinical perspective as, when injected into LA areas, insulin has much higher chances to reach into the subcutaneous muscle tissue, thus causing severe hypoglycemic events. An even rarer type of lipodystrophy is amyloidosis, ${ }^{9-12}$ for which a biopsy and pathologic diagnosis is often necessary. Insulin injected into areas of amyloidosis may suffer from significantly impaired absorption. LH generally regresses soon after the cessation of insulin injection, whereas localized amyloidosis does not. Distinguishing these lesions is clinically important.

Taking into consideration all above mentioned concepts, LD does not seem to represent only a complication of insulin treatment: a number of different clinical pictures have been reported as well to be associated with skin lesions going by the name of "LD". This of course causes confusion.

Indeed, in these case a major confounding factor depends of the term "lipodystrophy" itself. In fact, clinicians working in fields other than diabetes have been describing for many years LDs as heterogeneous, genetic or acquired disorders characterized by selective loss of body fat and predisposition to insulin resistance per se. ${ }^{13-18}$
In this case the extent of fat loss determines the severity of mostly associated metabolic complications, including hypertriglyceridemia, liver steatosis and, again, intriguingly enough, diabetes mellitus. The autosomal recessive congenital generalized lipodystrophy (CGL) and autosomal dominant familial partial lipodystrophy (FPL) are the two most common types of genetic LDs. AGPAT2, BSCL2, CAV1, and PTRF mutations have been reported in CGL, as LMNA, PPARG, AKT2, and PLIN1 mutations have been in FPL. Furthermore, CIDEC has been recognized as the disease gene for autosomal recessive FPL while LMNA and ZMPSTE24 have been so for autosomal recessive, mandibulo-acral dysplasia-associated lipodystrophy. An autosomal recessive auto-inflammatory lipodystrophy syndrome has been recently described and reported to depend on PSMB8 mutation. Molecular bases of many rare forms of genetic lipodystrophy remain to be elucidated. The acquired generalized and partial LDs are mainly autoimmune in origin and display complement abnormalities while localized LDs depend on drug or vaccine injections, pressure, panniculitis, and other unknown reasons. ${ }^{19}$ Treatment encompasses cosmetic surgery, as well as, early identification and medical treatment of metabolic/other complications based on diet, exercise, hypoglycemic drugs, and lipid-lowering agents.

The most prevalent subtype of acquired non-insulin treatment related lipodystrophy occurs in HIV-infected patients with longlasting protease inhibitor-containing, highly-active antiretroviral therapy. Also in these cases LD is commonly represented by its two main clinical features, LH and LA, probably due to disorders of lipid and/or glucose metabolism, the latter ranging from altered fasting hyperglycemia to insulin resistance/hyperinsulinemia. ${ }^{20,21}$ Recent studies on fatty acid metabolism and growth hormone dynamics have helped elucidate some still ill-defined, yet patho-genetically relevant aspects of HIV-associated lipohypertrophy and opened the door to new therapeutic interventions. One of these relies on tesamorelin, an analog of growth hormone-releasing hormone (GHRH) which appears to be active against visceral fat accumulation and to improve lipid parameters with minimal adverse effects on glucose tolerance. At the moment anyway dosing of tesamorelin still awaits better definition both per se and in view of long-term metabolic and cardiovascular outcomes. 22

Another reversible LD association reported to date is the one between LH and pegvisomant injections in patients with acromegaly. Several cases of such association have been published ${ }^{23}$ together with other documented side effects reported in the initial registration studies including headache, injection-site reactions, flu-like syndrome, and reversible elevation of hepatic enzymes. Interestingly enough, LH regressed in all patients when the medication was discontinued or a constant rotation of injections sites was implemented after a structured educational program. ${ }^{24}$

The analysis of the clinical forms of lipohypertrophy allows us to draw some conclusions. The term of lipohypertrophy is associated with many and various clinical pictures, although the most common form is a complication of insulin therapy, probably due to the great number of insulin-treated subjects with diabetes. Other injectables can cause LH, including pegvisomant for acromegaly treatment. In both conditions the educational aspects are critical both to identify LH by an appropriate methodology and to have patients implementing proper injection site rotation. In this brief review we provide an example of how the expertise and training of health care personnel is crucial to routinely look for and identify LH areas early enough in case of easily underdiagnosed lesions. ${ }^{25}$ 
However, the presence of other forms of LH, either genetic or related to HIV infection, still remains: they are difficult to explain but often linked to disorders of glucose metabolism, and defects in insulin sensitivity/production. This obviously represents a nomenclature problem and warrants clarification in the next future, because conditions known to be so different from each other need to be more accurately defined in order to better elucidate their inner mechanisms and thus provide clinicians with the best possible treatment strategies.

\section{Acknowledgments}

None.

\section{Conflict of interest}

Author declares that there is no conflict of interest.

\section{References}

1. AlAjlouni M,Abujbara M, Batieha A, et al. Prevalence of lipohypertrophy and associated risk factors in insulin-treated patients with type 2 diabetes mellitus. Int J Endocrinol Metab. 2015;13(2):e20776.

2. Blanco M, Hernández MT, Strauss KW, et al. Prevalence and risk factor of lipohypertrophy in insulin-injecting patients with diabetes. Diabetes Metab. 2013;39:445-453.

3. Forum for Injection Technique \& Therapy Expert Recommendations (FITTER). 2015.

4. Gentile S, Guarino G, Guida P, et al. A suitable palpation technique allows to identify skin lipohypertrophic lesions in insulin-treated people with diabetes. SpringerPlus; 2016.

5. Gentile S, Agrusta M, Guarino G, et al. Metabolic consequence of incorrect insulin administration techniques in aging subjects with diabetes. Acta Diabetol. 2011;48(2):121-125.

6. Grassi G, Scuntero P, Trepiccioni R, et al. Optimizing insulin injection technique and its effect on blood glucose control. Journal of Clinical and Translational Endocrinology. 2014;1(4):145-150.

7. Definitions from Medline Plus, an online service of the US National Institutes of Health (NIH)

8. Fujikura J, Fujimoto M, Yasue S, et al. Insulin-induced lipohypertrophy: report of a case with histopathology. Endocr J. 2005;52(5):623-628.

9. Nagase T, Katsura Y, Iwaki Y, et al. The insulin ball. Lancet. 2009;373(9658):184

10. Okamura S, Hayashino Y, Kore-Eda S, et al. Localized Amyloidosis at the Site of Repeated Insulin Injection in a Patient With Type 2 Diabetes Diabetes Care. 2013;36(12):e200.
11. Nagase T, Iwaya K, Iwaki Y, et al. Insulin-derived Amyloidosis and Poor Glycemic Control: A Case Series. Am J Med. 2014;127(5):450-454.

12. Swift B, Hawkins PN, Richards C, et al. Examination of insulin injection sites: an unexpected finding of localized amyloidosis. Diabet Med. 2002;19(10):881-886.

13. Rodriguez AJ, Mastronardi CA, Paz-Filho GJ. New advances in the treatment of generalized lipodystrophy: role of metreleptin. Ther Clin Risk Manag. 2015;11:1391-1400.

14. Nolis T. Exploring the pathophysiology behind the more common genetic and acquired lipodystrophies. J Hum Genet. 2014;59(1):16-23.

15. Fardet L, Vigouroux C, Capeau J. Lipodystrophies. Rev Med Interne. 2013;34(10):614-622.

16. Capeau J, Magré J, Caron-Debarle M, et al. Human lipodystrophies: genetic and acquired diseases of adipose tissue. Endocr Dev 2010;19:1-20

17. Garg A. Clinical review: Lipodystrophies: genetic and acquired body fat disorders. J Clin Endocrinol Metab. 2011;96(11):3313-3325.

18. Garg A. Lipodystrophies. Am J Med. 2000;108(2):143-152.

19. Richardson T, Kerr D. Skin-related complications of insulin therapy: epidemiology and emerging management strategies. Am J Clin Dermatol. 2003;4(10):661-667.

20. Kumar NS, Shashibhushan J, Malappa, et al. Lipodystrophy in Human Immunodeficiency Virus (HIV) Patients on Highly Active Antiretroviral Therapy (HAART). J Clin Diagn Res. 2015;9(7):OC05-OC08.

21. Leung VL, Glesby MJ. Pathogenesis and treatment of HIV lipohypertrophy. Curr Opin Infect Dis. 2011;24(1):43-49.

22. Rochira V, Zirilli L, Diazzi C, et al. Clinical and radiological evidence of the recurrence of reversible pegvisomant-related lipohypertrophy at the new site of injection in two women with acromegaly: a case series. $J$ Med Case Rep. 2012;6:2.

23. Bonert VS, Kennedy L, Petersenn S, et al. Lipodystrophy in patients with acromegaly receiving pegvisomant. $J$ Clin Endocrinol Metab. 2008;93(9):3515-3518.

24. Marazuela M, Daudén E, Ocón E, et al. Pegvisomant-induced lipohypertrophy: report of a case with histopathology. Ann Intern Med 2007; 147(10):741-743.

25. Holstein A, Stege H, Kovacs P. Lipoatrophy associated with the use of insulin analogues: a new case associated with the use of insulin glargine and review of the literature. Expert Opin Drug Saf. 2010;9(2):225-231. 